\title{
CubeSat Material Limits For Design for Demise
}

\author{
R. L. Kelley ${ }^{1}$, D. R. Jarkey ${ }^{2}$ \\ 1. Jacobs, NASA Johnson Space Center, Houston, TX 77058, USA \\ 2. HX5 - Jacobs JETS Contract, NASA Johnson Space Center, Houston, TX 77058, USA
}

The CubeSat form factor of nano-satellite (a satellite with a mass between one and ten kilograms) has grown in popularity due to their ease of construction and low development and launch costs. In particular, their use as student led payload design projects has increased due to the growing number of launch opportunities. CubeSats are often deployed as secondary or tertiary payloads on most US launch vehicles or they may be deployed from the ISS. The focus of this study will be on CubeSats launched from the ISS.

From a space safety standpoint, the development and deployment processes for CubeSats differ significantly from that of most satellites. For large satellites, extensive design reviews and documentation are completed, including assessing requirements associated with reentry survivability. Typical CubeSat missions selected for ISS deployment have a less rigorous review process that may not evaluate aspects beyond overall design feasibility. CubeSat design teams often do not have the resources to ensure their design is compliant with reentry risk requirements.

A study was conducted to examine methods to easily identify the maximum amount of a given material that can be used in the construction of a CubeSats without posing harm to persons on the ground. The results demonstrate that there is not a general equation or relationship that can be used for all materials; instead a limiting value must be defined for each unique material. In addition, the specific limits found for a number of generic materials that have been previously used as benchmarking materials for reentry survivability analysis tool comparison will be discussed. 